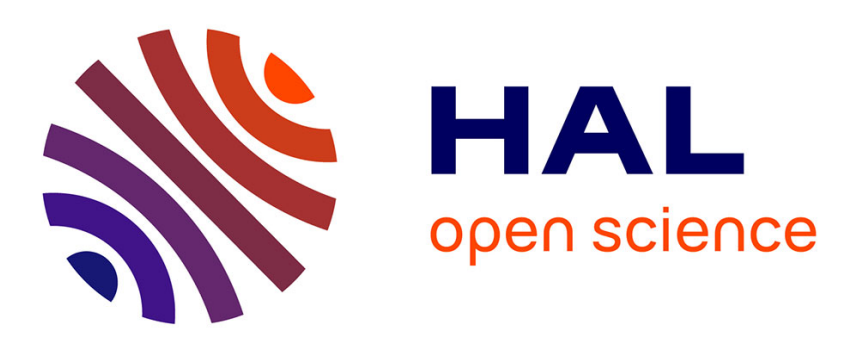

\title{
Guard Time Optimisation and Adaptation for Energy Efficient Multi-hop TSCH Networks
}

Georgios Papadopoulos, Alexandros Mavromatis, Xenofon Fafoutis, Nicolas Montavont, Robert Piechocki, Theo Tryfonas, George Oikonomou

\section{- To cite this version:}

Georgios Papadopoulos, Alexandros Mavromatis, Xenofon Fafoutis, Nicolas Montavont, Robert Piechocki, et al.. Guard Time Optimisation and Adaptation for Energy Efficient Multi-hop TSCH Networks. WF-Iot 2016 : 3rd IEEE World Forum on Internet of Things, Dec 2016, Reston, Va, United States. pp.301 - 306, 10.1109/WF-IoT.2016.7845475 . hal-01609086

\section{HAL Id: hal-01609086 https://hal.science/hal-01609086}

Submitted on 3 Oct 2017

HAL is a multi-disciplinary open access archive for the deposit and dissemination of scientific research documents, whether they are published or not. The documents may come from teaching and research institutions in France or abroad, or from public or private research centers.
L'archive ouverte pluridisciplinaire HAL, est destinée au dépôt et à la diffusion de documents scientifiques de niveau recherche, publiés ou non, émanant des établissements d'enseignement et de recherche français ou étrangers, des laboratoires publics ou privés. 


\title{
Guard Time Optimisation and Adaptation for Energy Efficient Multi-hop TSCH Networks
}

\author{
Georgios Z. Papadopoulos*, Alexandros Mavromatis ${ }^{\dagger}$, Xenofon Fafoutis ${ }^{\dagger}$, \\ Nicolas Montavont*, Robert Piechocki ${ }^{\dagger}$, Theo Tryfonas ${ }^{\dagger}$ and George Oikonomou ${ }^{\dagger}$ \\ *IRISA, Télécom Bretagne, Institut Mines-Télécom, France \\ \{georgios.papadopoulos, nicolas.montavont\}@telecom-bretagne.eu \\ ${ }^{\dagger}$ Faculty of Engineering, University of Bristol, UK \\ \{a.mavromatis, xenofon.fafoutis, r.j.piechocki, g.oikonomou, theo.tryfonas\}@bristol.ac.uk
}

\begin{abstract}
In the IEEE 802.15.4-2015 standard, Time Slotted Channel Hopping (TSCH) aims to guarantee high-level network reliability by keeping nodes time-synchronised. In order to ensure successful communication between a sender and a receiver, the latter starts listening shortly before the expected time of a MAC layer frame's arrival. The offset between the time a node starts listening and the estimated time of frame arrival is called guard time and it aims to reduce the probability of missed frames due to clock drift. In this paper, we investigate the impact of the guard time on network performance. We identify that, when using the 6tisch minimal schedule, the most significant cause of energy consumption is idle listening during guard time. Therefore, we first perform mathematical modelling on a TSCH link to identify the guard time that maximises the energy-efficiency of the TSCH network in single hop topology. We then continue in multi-hop network, where we empirically adapt the guard time locally at each node depending its distance, in terms of hops, from the sink. Our performance evaluation results, conducted using the Contiki OS, demonstrate that the proposed decentralised guard time adaptation can reduce the energy consumption by up to $40 \%$, without compromising network reliability.
\end{abstract}

Index Terms-Internet of Things, IEEE 802.15.4-2015, TSCH, Synchronisation, Guard Time, Energy Consumption.

\section{INTRODUCTION}

In 2016 the IEEE 802.15.4-2015 standard [1] was published to offer a certain quality of service for deterministic industrialtype applications. Among the operating modes defined in this standard, Time-Slotted Channel Hopping (TSCH) is a Medium Access Control (MAC) protocol for low-power and reliable networking solutions in Low-Power Lossy Networks (LLNs). Although there is a vast literature of unstandardised MAC protocols that are optimised for different scenarios [2], [3], the standardised TSCH offers interoperability between IoT devices. Indeed, at its core, TSCH implements a channel hopping scheme to avoid interference, and consequently to enable high reliability [4], while it employs time synchronisation to achieve low-power operation, see Fig. 1. TSCH presents a deterministic scheduling approach where each cell consists a pair of timeslot and channel offset for collision avoidance purposes. Each channel offset is translated into a frequency as follows:

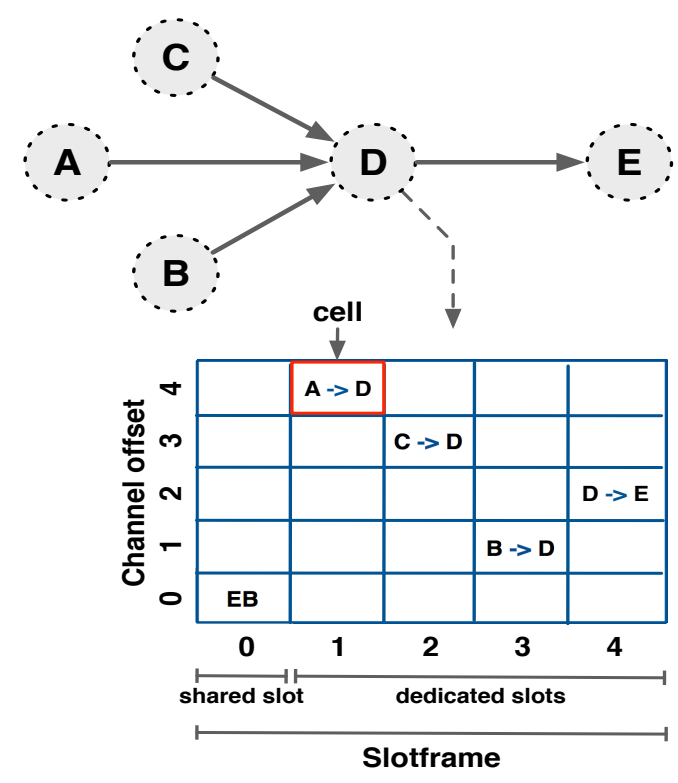

Fig. 1. An example TSCH schedule for node D. $A \rightarrow D$ stands for "node A transmits to node D", while $E B$ cells are used for broadcast and advertising control packets.

$$
\text { frequency }=F\{(A S N+\text { channelOffset }) \% n F r e q\},
$$

where A.SN is the Absolute Sequence Number, while $n$ Freq is the number of available frequencies (e.g., 16 when using IEEE 802.15.4-compliant radios at $2.4 \mathrm{GHz}$ with all channels in use) [5].

Continuously re-synchronizing the network may prevent the loss of synchronization. Thus, to account for loss of synchronisation, under TSCH scheme, a receiver maintains its radio on receiving mode for an extended period of time, named Guard Time. More specifically, the receiver turns its radio on slightly before the scheduled transmission to guarantee the successful packet reception, see Fig. 2. In [6], [7], we highlighted the effect of guard time on single hop network. Indeed, we identified that, when employing the 6tisch minimal schedule in point to point link, most of the energy consumed is wasted in idle listening, due to the guard time. 
In this paper, we further investigate the importance of guard time in a multi-hop network. In multi-hop TSCH networks, the guard time is typically configured statically and, therefore, needs to account for the worst case scenario, not only in terms of clock drift, but also in terms of network size (see, for example, the Contiki OS $^{1}$ [8] and OpenWSN [9] implementations of the TSCH protocol). Instead, in this work, we demonstrate that TSCH could significantly improve their energy-efficiency by adapting their guard time in an independent and decentralised manner. To this aim, we first study the optimal guard time values as a function of the clock drift, using both an analytical model and simulations. We then present our thorough empirical study for decentralised guard time adaptation for energy-efficient multi-hop TSCH networks. Our performance evaluation results on top of COOJA (a simulator for Contiki OS), demonstrate that fine-tuning the guard time on each node can significantly improve the energy efficiency of a TSCH multi-hop network without compromising its reliability.

The remainder of our paper is organised as follows. Section II provides an overview of the TSCH mechanics and summarises the related work. We then detail our problem formulation and we present guard time optimisations in Section II proposing a mechanics for adaptive guard time adaptation in multi-hop TSCH networks. We implement our solutions on top of the Contiki OS and then demonstrate their performance in Section IV, focusing on the following metrics: energy consumption and reliability. Finally, Section V provides the concluding remarks and future perspectives for our work.

\section{TSCH MECHANICS AND RELATED WORK}

In TSCH networks, time is divided into timeslots of equal length, large enough to transmit a frame and to receive an acknowledgement, while a set of timeslots construct a slotframe. At each timeslot, a node may transmit or receive a frame, or it may turn its radio off for saving energy. Each timeslot can be either dedicated (contention-free) or shared (contention-based approach). Finally, each timeslot is labelled with ASN, a variable which counts the number of timeslots since the network was established, ASN is initialised to 0. Fig. 2 illustrates a typical TSCH-based communication between two nodes.

Since the clock of each node drifts relative to its neighbours', nodes periodically exchange Enhanced Beacon (EB) packets to periodically re-synchronise, and thus, to remain time-synchronised throughout the deployment's lifetime. Synchronisation does not need explicit EB exchange, data packets may also be utilised to compute clock drifts [10]. Typically, an EB contains the current ASN and channel frequency information, as well as information about the initial link and slotframe for new nodes to join the network. New nodes may join a TSCH network by "hearing" an EB frame from another node. In $\mathrm{TSCH}$, there are two ways for two nodes to re-synchronise, frame-based and acknowledgment-based synchronisation, respectively [11]. During the re-synchronisation

\footnotetext{
${ }^{1}$ Contiki OS - www.contiki-os.org
}

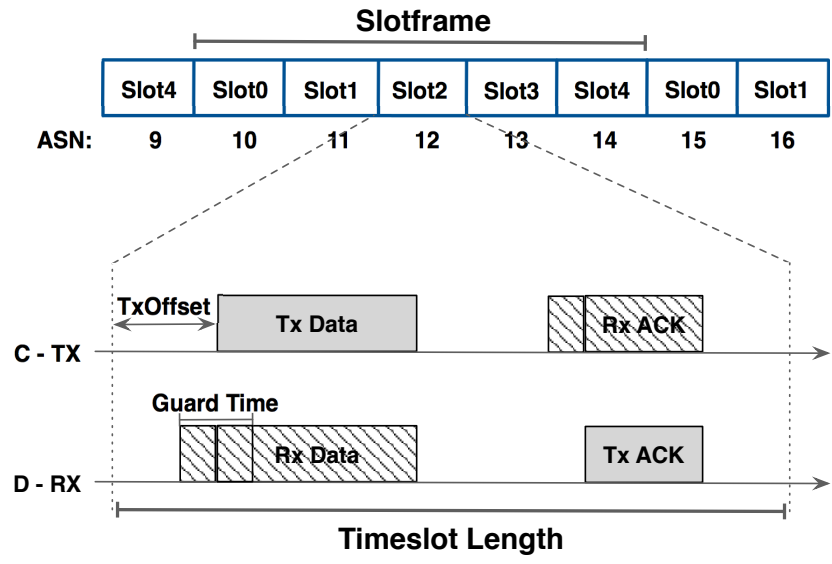

Fig. 2. A typical TSCH timeslot template for a transmitter (top) and receiver node (bottom): node $\mathrm{C}$, transmits its data packet after TxOffset, while the receiver $\mathrm{D}$, uses a Guard Time to avoid missing the incoming packet by turning its radio on slightly before the packet arrival.

process, the receiver node calculates the offset, the relative de-synchronisation time. In frame-based synchronisation, the offset is applied at the receiver side, while in ACK-based, it is applied to the sender's timing.

Specifically in [12] the authors suggest that in a $\mathrm{TSCH}$ network the resynchronisation frequency should be reduced while the nodes in the network are synchronised. In order to keep synchronisation the nodes measure the time they need to drift relatively to their neighbour node. Then nodes periodically make a time correction based on the previews calculated period. This frequency reduction results to a lower duty cycle and a longer network lifetime.

In [13], the authors present an adaptive compensation algorithm that allows synchronisation between sensor nodes in Wireless Sensor Network (WSN), without exchanging any control or data packet. To do so, each node averages the clock offset in a given period, thus the proposed scheme compensates the clock drift error by periodically adjusting the local clock of each node in order to improve the accuracy of the clock drift. However, it requires that the time synchronisation error will not fall out of the guard time boundaries so the node will be able to adjust its clock.

The idea of implementing an adaptive logic in the TSCH schedule has been proposed many times. Concretely we use a multi-hop topology to put our suggested methodology to the test. Likewise the technique presented in [10] is tested also in a multi-hop topology where the author suggests an adaptive synchronisation technique in order to keep a TSCH network synchronised. The core of this technique is that nodes will predict how their clock will be drifting relatively to their neighbours. Therefore the node time stamps the instants and resynchronises at that state. The later will be accomplished through software by adding a field to the EB and ACK packets, the field will have the information about the time that the neighbour node synchronised, and right after it will synchronise to. 


\section{GUARD TIME AdAPTATION}

In this paper, we propose the decentralised adaptation of the guard time for reducing the energy-consumption of a TSCH network. To this end, we begin with the analysis of a $\mathrm{TSCH}$ link, demonstrating that there is a guard time that maximises its energy-efficiency.

\section{A. Guard Time Optimisation in a TSCH link}

A node transmits a data packet at the beginning of each timeslot, exactly after the TxOffset. TSCH incorporates a Guard Time to account for clock drift. Indeed, to account for both positive and negative clock drift, the receiver wakes up before the expected end of the TxOffset and keeps the radio on for $\tau$ seconds or until a frame preamble is received. The guard time $\tau$ is equally spaced around the end of the TxOffset. Thus, for a certain guard time, $\tau$, the maximum synchronisation error, $\epsilon_{\tau}$, that can be tolerated is:

$$
\epsilon_{\tau}=\frac{\tau}{2}-\tau_{p}
$$

where $\tau_{p}$ is the time required for the reception of the frame preamble. Let us consider the use of clocks with an error of $\pm e_{f}$. The synchronisation error accumulates over time. The worst case scenario for synchronisation is right before a synchronisation event (e.g., EB frame), when the error is:

$$
\epsilon_{T}=T\left(\frac{1}{1-e_{f}}-\frac{1}{1+e_{f}}\right),
$$

where $T$ is the period of synchronisation events. By equating (2) and (3), we calculate a minimum guard time required to achieve zero packet loss due to loss of synchronisation $\left(\tau_{m}\right)$ :

$$
\tau_{m}=2 T\left(\frac{1}{1-e_{f}}-\frac{1}{1+e_{f}}\right)+2 \tau_{p} .
$$

Observe that in the ideal case where the clock error is $e_{f}=0$ $\mathrm{ppm}$, the minimum acceptable guard time is $\tau_{m}=2 \tau_{p}$.

The minimum guard time, given by (4), constitutes the optimum value for achieving the highest energy-efficiency in the TSCH link. Indeed, longer guard times would lead in unnecessary time spent in idle listening, whilst shorter guard time would lead into packet loss due to loss of synchronisation, i.e., energy wasted for the transmission of packets that do not reach their destination.

\section{B. Guard Time Adaptation in Multi-Hop TSCH Networks}

In a multi-hop context, the clock drift increases with the width of the network. As a result, a longer guard time is required to maintain synchronisation. Similarly to a single-hop link, there is a network-wide optimum guard time configuration, that is the minimum guard time that keeps the network synchronised. Yet, as we show in the simulations that follow, the network-wide optimum guard time, is not necessarily the optimum value for each individual node. Hence, the energy consumption of the TSCH network can be further improved by adapting the guard time in a decentralised manner.
TABLE I

SimULATION SETUP.

\begin{tabular}{ll}
\hline \hline Topology & Value \\
\hline Topology & 1-hop \\
& Multi-hop \\
Number of nodes & 1-hop: 2 (a transmitter and receiver) \\
& Multi-hop: 2 to 10 (including the sink) \\
1-hop: $20 \mathrm{~m}$ in line \\
Node spacing & Multi-hop: $100 \mathrm{~m}$ in line \\
& Value \\
\hline Simulation & 60 minutes \\
\hline Duration & 1 pkt $/ 60 \mathrm{sec}$ \\
Traffic Pattern & 102 bytes $(77$ bytes payload $)$ \\
Data packet size & RPL [14] \\
Routing model & TSCH $(6 \mathrm{TiSCH}$ minimal schedule) \\
MAC model & Value \\
\hline TSCH & $3.42 \mathrm{~s}$ \\
\hline EB frequency & 7 \\
Slotframe length & $15 \mathrm{~ms}$ \\
Timeslot length & $(0-2200) \mu \mathrm{s}$ \\
Guard Time & $1-h o p:(0, \pm 10, \pm 20, \pm 30, \pm 40) \mathrm{ppm}$ \\
Clock Drift & Multi-hop: \pm 20 \\
& Value \\
\hline Hardware & CC2420 \\
\hline Antenna model & $2.4 \mathrm{GHz}$ \\
Radio propagation & $0 \mathrm{dBm}$ \\
Transmission power & \\
\hline \hline
\end{tabular}

We follow a heuristic approach to investigate the network behaviour under various guard times. As shown in the simulations that follow, our exhausted study demonstrates that the optimum guard time essentially depends on the number of hops away from the sink. Hence, the performance of the TSCH network can be improved by adapting each individual node's guard time to its hop-distance from the sink node. To this aim, we begin by empirically identifying and constructing a look-up table of the minimum guard times that maintain synchronisation in various hop-distances from the sink. In runtime, each node then adapts its guard time using the lookup table and information obtained from the routing protocol regarding its hop-distance to the sink.

\section{Performance evaluation}

In order to evaluate the impact of guard time in the energy consumption of TSCH network and the benefits of guard time adaptation, we conducted a large set of simulations by employing COOJA, the network simulator distributed as part of the Contiki OS, and emulating the Z1 motes. Hereafter, we present a thorough performance evaluation for both statically configured and our adaptive scheme. The details of the simulation setup are presented in Table I.

\section{A. Single-hop TSCH Links:}

1) Setup: We first focused on single-hop $\mathrm{TSCH}$ links that employ the 6tisch mininum schedule. We studied the behaviour of TSCH links under various realistic clock drifts (i.e., $\pm 10, \pm 20, \pm 30$ and $\pm 40 \mathrm{ppm}$ ). Indeed, we considered the worst case scenario TSCH link, thus, we configured the transmitter node to the maximum positive clock drift and the receiver at the maximum negative drift. For instance, in the case of the $\pm 20 \mathrm{ppm}$ configuration, we set the transmitter node at $+20 \mathrm{ppm}$ and the receiver at $-20 \mathrm{ppm}$, resulting to 


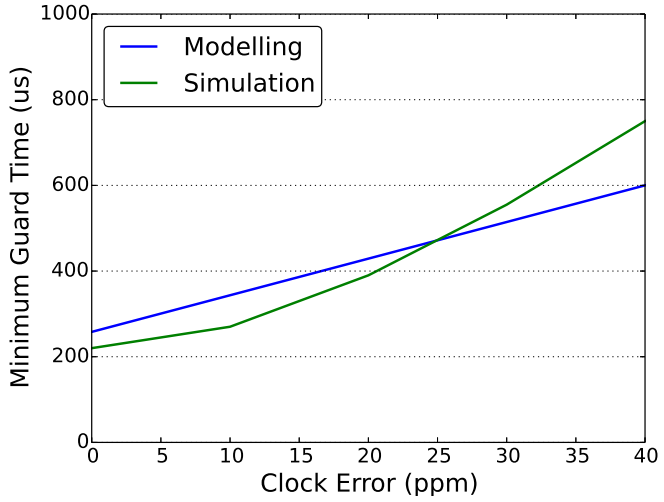

(a) Minimum guard time for operation without packet loss.

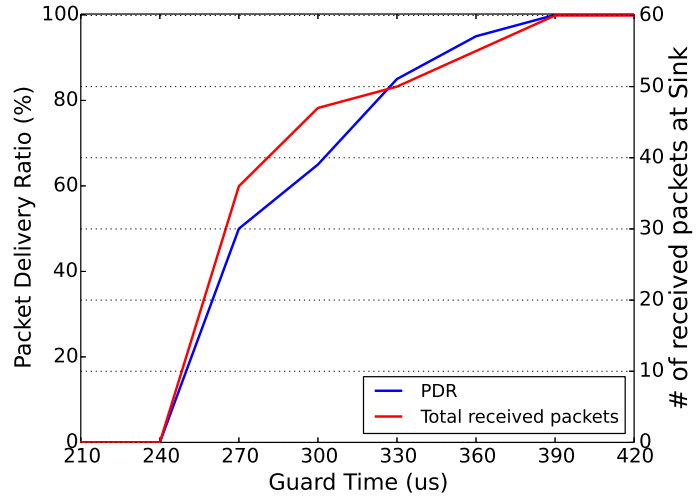

(b) PDR and goodput under \pm 20 ppm clock drift.

Fig. 3. Minimum required guard time for various clock drifts (left) and the network performance under $\pm 20 \mathrm{ppm}$ clock drift (right), values are in average.

a relative drift of $40 \mathrm{ppm}$. Note that the clock drifts are constant throughout each simulation. Furthermore, we conducted simulations under various guard time (e.g., 400, 600, $800 \mu \mathrm{s})$ configurations, while keeping the default values for the rest of parameters such as EB or data packet transmission frequency.

We have conducted a scenario with two nodes, leaf transmitter and sink receiver, respectively, positioned at a distance of $20 \mathrm{~m}$. We choose the data packet size to be equal to 102 bytes that corresponds to all necessary information for MAC, routing and application operations. Furthermore, we set our network to run with the Unit Disk Graph Medium (UDGM) for the sake of clarity, where each node emits at $0 \mathrm{dBm}$ transmission power. Finally, each simulation lasted $60 \mathrm{~min}$.

In [6], we studied the impact of the idle listening during guard time on energy consumption. Hereinafter, we demonstrate the guard time optimisation that we performed, as well as the gains of the fine-tuning TSCH configurations in terms of reliability, goodput and energy consumption in single-hop communication.

2) Guard Time: We first investigate the minimum guard time that guarantees $100 \%$ Packet Delivery Ratio (PDR), under different clock drift values (i.e., $0, \pm 10, \pm 20, \pm 30$ and \pm $40 \mathrm{ppm}$ ) using both the analytical model, presented in the previous Section, and a simulation campaign. Note that packet loss is calculated as $1-P D R$, and thus, packet loss $0 \%$ is the equivalent of 100\% PDR. As can be observed from Fig. 3a, our model (i.e., Eq. (4)) approximates a linear behaviour between the guard time and clock error, when $\tau_{p}=129 \mu \mathrm{s}$ and $T=1.71 \mathrm{~s}$. Indeed, the larger is the clock drift, the longer the required minimum guard time for guaranteeing $100 \%$ of link reliability. It is important to mention that our simulation results validate our mathematical analysis, since they present almost a linear performance. Note that both nodes operate as EB transmitters and receivers; thus, the link is synchronised at half the EB period on average, $T=3.42 / 2=1.71 \mathrm{~s}$.

To further validate our empirical study, we here focus in the case of $\pm 20 \mathrm{ppm}$, a typical worst-case clock drift in IoTdevices [15]. Our performance evaluation results demonstrate that $390 \mu \mathrm{s}$ is the minimum guard time length for efficient $\mathrm{TSCH}$ operation without compromising network reliability due
TABLE II

Approximate Current Consumption of Z1 Mote.

\begin{tabular}{llr}
\hline \hline IC & Notes & Current Consumption \\
\hline CC2420 & TX Mode @ 0 $\mathrm{dBm}$ & $17.4 \mathrm{~mA}$ \\
& RX Mode & $18.8 \mathrm{~mA}$ \\
MSP430f2617 & $4 \mathrm{~mA}$ \\
& Active Mode @ 8 $\mathrm{MHz}$ & $0.5 \mathrm{uA}$ \\
\cline { 2 - 2 }
\end{tabular}

to loss of synchronisation. Indeed, as can be seen from Fig. 3b, only above $390 \mu$ s of guard time, both the reliability and the goodput, the application-level throughput (i.e., the number of actual data packets delivered to the sink), achieve $100 \%$ of performance.

3) Energy Efficiency: To evaluate the energy consumption of each node in the network, we employed the Contiki's Powertrace and Energest power profile to estimate power consumption. This module monitors and logs in realtime the radio and Central Processing Unit (CPU) usage by tracking the time spent in each state (i.e., transmitting, receiving, awaken, sleeping). Table II provides the current consumption levels at each of these states for $\mathrm{Z} 1$ mote $^{2}$, considering that it is powered at $3 \mathrm{~V}$.

We here investigate the impact of the guard time on energy consumption, under $\pm 20 \mathrm{ppm}$ clock drift. To this aim, we first present energy consumption performance under various guard time configurations. Our results demonstrate that by reducing the guard time (i.e., from $2200 \mu \mathrm{s}$, default configuration of Contiki's TSCH implementation, to $400 \mu \mathrm{s}$ ), we can decrease the average power consumption per node (i.e., receiver node in our scenario) by more than $40 \%$, see Fig. 4a. Indeed the energy consumption is reduced further at guard times of less than $390 \mu \mathrm{s}$, yet at the cost of compromising the network reliability. To better visualise this trade-off, we define the energy-efficiency metric of TSCH as the average energy consumed for the successful reception of a single bit, and it is calculated as follows:

$$
\eta=\frac{E}{P D R * T_{\text {transmissions }} * P_{\text {size }} * 8},
$$

\footnotetext{
${ }^{2}$ http://zolertia.sourceforge.net/wiki/images/e/e8/Z1_RevC_Datasheet.pdf
} 


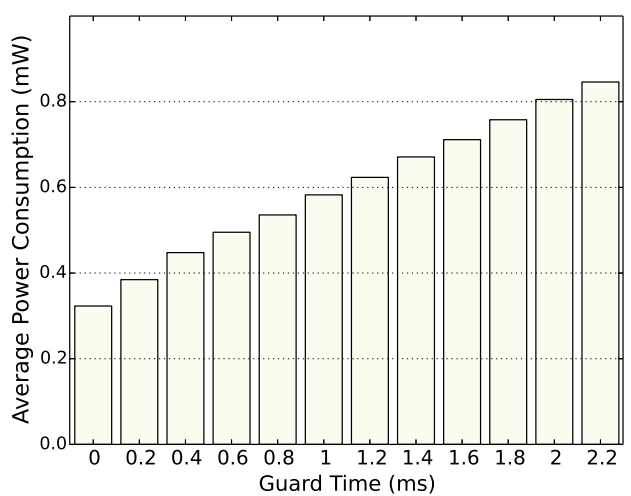

(a) Average power consumption, under various guard time lengths.

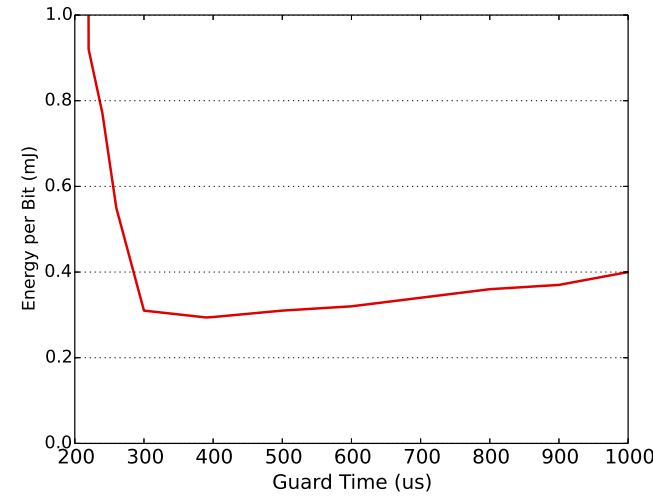

(b) Energy-Efficiency: Energy consumption per successful received bit.

Fig. 4. A thorough power consumption performance of the TSCH scheme, under \pm 20 ppm clock drift [15].

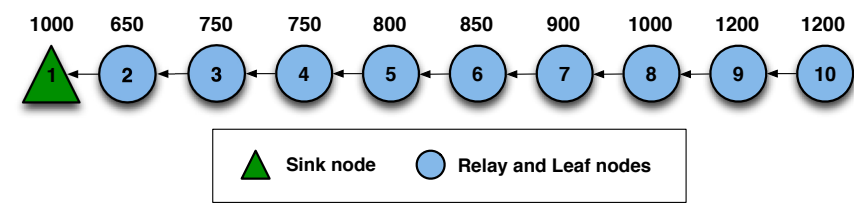

Fig. 5. The analysed adaptive multi-hop scenario of 10 nodes, including the sink, topology: each node is fine-tuned at the corresponded minimum required guard time according to its distance from the sink.

where $E$ is the total energy consumed during the experiment, $T_{\text {transmissions }}$ is the total packet transmissions from the leaf to the sink node, while $P_{\text {size }}$ is the size of a data packet in bytes. Fig. $4 \mathrm{~b}$ plots the energy efficiency of TSCH as a function of the guard time. It can be observed that there is an optimisation point for the guard time at $390 \mu$ s. Below that optimal configuration the energy per correct bit increases rapidly, due to packet loss caused by loss of synchronisation. Above that optimal configuration the energy per correct bit increases again, as the energy consumed in idle listening increases with the guard time.

\section{B. Multi-Hop TSCH Networks}

We next consider multi-hop TSCH networks that employ the 6tisch minimal schedule, and evaluate the proposed guard time adaptation. To this aim, we performed large set of simulations to under different topologies. More specifically, we gradually increased the size of the multi-hop network, from single-hop up to 9 hops with 10 nodes that are positioned in line of $100 \mathrm{~m}$. This topology is presented in Fig. 5. Furthermore, we configured each node to transmit one data packet every 60 seconds. By employing the RPL protocol [14], each node is able to construct a Directed Acyclic Graph (DAG). Similarly, to the single-hop scenario, we consider a worst case scenario for synchronisation, in which all odd nodes have positive clock drift and all even nodes have negative clock drift. Finally, each simulation lasted $60 \mathrm{~min}$.

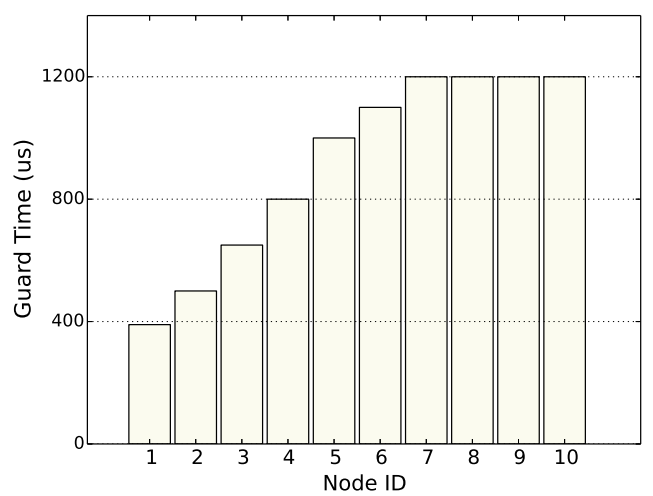

Fig. 6. Minimum required guard time for operation without packet loss due to loss of synchronisation, in 9 different multi-hop network topologies.

We first perform a large set of simulations for different topologies in order to estimate the minimum guard time that maintains $100 \%$ PDR. More specifically, we first set a single hop communication with two nodes to cross validate the previously presented mathematical analysis. Then we gradually extended our topology to multi-hop, from 2 to 9-hops in line, ending with the topology shown in Fig. 5. Our performance evaluation results demonstrate that nodes closer to the sink require less guard time, while from 7th hop this value is stabilised at $1200 \mu \mathrm{s}$. The empirically-derived minimum required guard time for each hop is illustrated in Fig. 6, which is then used as a look-up table to adapt the guard time in run-time.

In this setup, the empyrically-derived network-wide optimum guard time is $1200 \mu \mathrm{s}$. We then compared our adaptive scheme against a statically and homogeneously configured (i.e., guard time at $1200 \mu \mathrm{s}$ ) multi-hop network. Our performance evaluation results demonstrate that by fine-tuning the guard time at each node according to its hop-distance to the sink, yields significant energy savings for the TSCH network. 


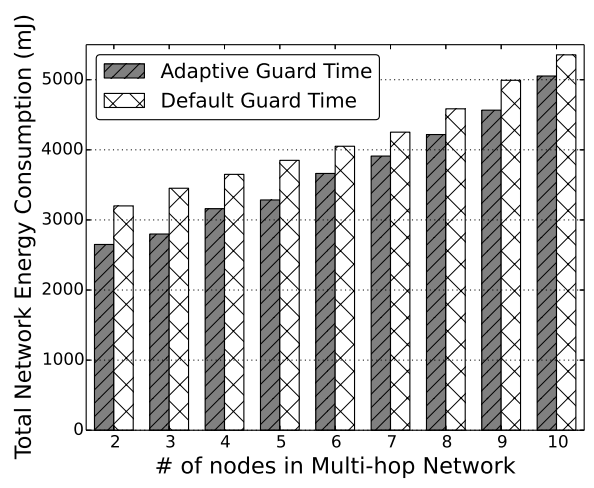

(a) Total energy consumption for adaptive and statically configured multi-hop networks, in 9 different scenarios (from 2-hops to 9-hops topology).

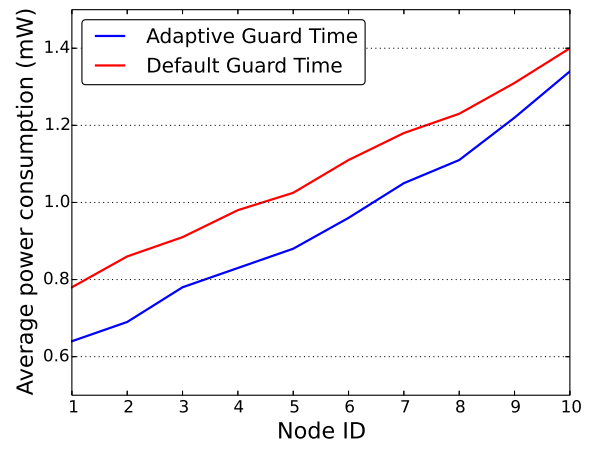

(b) Average energy consumption per hop node, in 9-hops scenario (node ID1 is the sink)

Fig. 7. The gain of guard time adaptation evaluated under 9 multi-hop topologies (in line), from 2-hops to 9-hops with nodes positioned in distance of $100 \mathrm{~m}$.

Indeed, our adaptive scheme essentially reduces the energy consumption for all the considered topologies by more than $12 \%$ in average, while up to $17 \%$ in 2-hop and 3-hop scenario, see Fig. 7a. Furthermore, as can be observed from Fig. 7b, the further away the nodes from the sink the less efficient is guard time adaptation approach in terms of energy consumption. Since the more away the node is from the sink, the more prone to packet losses, and thus, more retransmissions in the network are necessary.

\section{CONCLUSIONS}

In this work, we first investigated the impact of guard time on TSCH performance in terms of network reliability, goodput and energy consumption. We then performed empirical optimisations on guard time to maximise the energy-efficiency in a multi-hop TSCH network. Our performance evaluation results on top of COOJA (a simulator for Contiki OS), demonstrated that the guard time has a straightforward impact on reliability and on energy consumption. In particular, it is shown that adapting the guard time at each node according to its distance from the sink can result into significant savings in energy consumption without compromising the reliability of the network. Our ongoing work consists of further investigating this lead in random multi-hop topologies, where the clock drift and the large set of nodes may impact heavily the network synchronisation. Furthermore, we plan to study the behaviour of TSCH under realistic conditions by performing a set of experimental studies on FIT IoT-LAB testbed [16].

\section{ACKNOWLEDGEMENTS}

This work was partially performed under the SPHERE IRC funded by the UK Engineering and Physical Sciences Research Council (EPSRC), Grant EP/K031910/1.

\section{REFERENCES}

[1] "IEEE Standard for Low-Rate Wireless Personal Area Networks (LRWPANs)," IEEE Std 802.15.4-2015 (Revision of IEEE Std 802.15.42011), April 2016.

[2] G. Z. Papadopoulos, "Improving Medium Access in Dynamic Wireless Sensor Networks," Ph.D. dissertation, University of Strasbourg, 2015.
[3] X. Fafoutis, A. Di Mauro, M. D. Vithanage, and N. Dragoni, "ReceiverInitiated Medium Access Control Protocols for Wireless Sensor Networks," Comput. Networks, vol. 76, pp. 55-74, Jan. 2015.

[4] T. Watteyne, A. Mehta, and K. Pister, "Reliability Through Frequency Diversity: Why Channel Hopping Makes Sense," in Proceedings of the 6th ACM Symposium on Performance Evaluation of Wireless Ad Hoc, Sensor, and Ubiquitous Networks, 2009, pp. 116-123.

[5] T. Watteyne, M. Palattella, and L. Grieco, "Using IEEE 802.15.4e Time-Slotted Channel Hopping (TSCH) in the Internet of Things (IoT): Problem Statement," RFC 7554, 2015.

[6] A. Mavromatis, G. Z. Papadopoulos, X. Fafoutis, A. Elsts, G. Oikonomou, and T. Tryfonas, "Impact of Guard Time Length on IEEE 802.15.4e TSCH Energy Consumption," in Proceedings of the IEEE International Conference on Sensing, Communication and Networking (SECON), 2016

[7] G. Z. Papadopoulos, A. Mavromatis, X. Fafoutis, R. Piechocki, T. Tryfonas, and G. Oikonomou, "Guard Time Optimisation for Energy E ciency in IEEE 802.15.4-2015 TSCH Links," in Proceedings of the EAI International Conference on Interoperability in IoT (InterIoT), 2016.

[8] S. Duquennoy, B. Al Nahas, O. Landsiedel, and T. Watteyne, "Orchestra: Robust mesh networks through autonomously scheduled tsch," in Proceedings of the 13th ACM Conference on Embedded Networked Sensor Systems, ser. SenSys '15. New York, NY, USA: ACM, 2015, pp. 337350 .

[9] T. Watteyne, X. Vilajosana, B. Kerkez, F. Chraim, K. Weekly, Q. Wang, S. Glaser, and K. Pister, "Openwsn: a standards-based low-power wireless development environment," Transactions on Emerging Telecommunications Technologies, vol. 23, no. 5, pp. 480-493, 2012.

[10] T. Chang, T. Watteyne, K. Pister, and Q. Wang, "Adaptive Synchronization in Multi-hop TSCH Networks," Computer Networks, vol. 76, pp. 165-176, 2015.

[11] P. Thubert, "An Architecture for IPv6 over the TSCH mode of IEEE 802.15.4," draft-ietf-6tisch-architecture-10, June 2016.

[12] D. Stanislowski, X. Vilajosana, Q. Wang, T. Watteyne, and K. S. J. Pister, "Adaptive Synchronization in IEEE802.15.4e Networks," IEEE Transactions on Industrial Informatics, vol. 10, no. 1, pp. 795-802, 2014.

[13] T. Chang and Q. Wang, "Adaptive compensation for time-slotted synchronization in wireless sensor network," International Journal of Distributed Sensor Networks, vol. 2014, no. 9, 2014.

[14] T. Winter, P. Thubert, A. Brandt, J. Hui, R. Kelsey, P. Levis, K. Pister, R. Struik, J. Vasseur, and A. R., "RPL: IPv6 Routing Protocol for LowPower and Lossy Networks," RFC 6550, 2012.

[15] X. Fafoutis, B. Janko, E. Mellios, G. Hilton, R. S. Sherratt, R. Piechocki, and I. Craddock, "SPW-1: A Low-Maintenance Wearable Activity Tracker for Residential Monitoring and Healthcare Applications," in Proceedings of the EAI International Conference on Wearables in Healthcare (HealthWear), 2016.

[16] G. Z. Papadopoulos, A. Gallais, G. Schreiner, and T. Noel, "Importance of Repeatable Setups for Reproducible Experimental Results in IoT," in Proceedings of the 13th ACM International Symposium on Performance Evaluation of Wireless Ad Hoc, Sensor, and Ubiquitous Networks (PEWASUN), 2016. 